\title{
Data Acquisition in 4D Atomic Electron Tomography
}

Jihan Zhou ${ }^{1}$, Yongsoo Yang ${ }^{1}$, Yao Yang ${ }^{1}$, Colin Ophus $^{2}$, Fan Sun ${ }^{3}$, Andreas K. Schmid ${ }^{2}$, Hao Zeng ${ }^{3}$, Peter Ercius $^{2}$ and Jianwei Miao ${ }^{1 *}$

1. Dept. of Physics \& Astronomy and California NanoSystems Institute, Univ. of California, Los Angeles, CA 90095, USA.

2. Molecular Foundry, Lawrence Berkeley National Laboratory, Berkeley, CA 94720, USA.

3. Dept. of Physics, Univ. at Buffalo, Buffalo, NY 14260, USA.

* Corresponding author: miao@physics.ucla.edu

3D structural analysis is essential to understand the structure and property relationships of materials. The properties of many materials strongly depend on vacancies, defects, surface reconstructions, grain boundaries, stacking faults, dislocations and disorder [1-3]. However, those 3D atomic arrangements are not accessible to crystallography. Recently, a groundbreaking technique, termed atomic electron tomography (AET) [1,4-7], has been developed to determine the 3D structure of crystal defects and disordered materials without the assumption of crystallinity. Here, we add a new dimension (time) to AET (i.e. 4D-AET) to study early stage nucleation dynamics in solid-solid phase transitions [8]. We have developed state-of-art data acquisition for 4D-AET and validated the method by designing and conducting a consistency check experiment of FePt nanoparticles undergoing phase transitions. We annealed the nanoparticles at $520^{\circ} \mathrm{C}$ for 9 minutes in vacuum and acquired two independent, sequential tilt series of an FePt nanoparticle using an annular dark-field scanning transmission electron microscope. Figure 1 shows 3D atomic models ( $\mathrm{Pt}$ in blue and $\mathrm{Fe}$ in red) of the nanoparticle obtained from two independent experimental measurements and determined by AET. By comparing their 3D atomic coordinates, we confirmed that $95.4 \%$ of atoms are consistent between the two models and the precision of our 3D atomic structure determination method is $26 \mathrm{pm}$. Next, we trapped another FePt nanoparticle at different annealing times and acquired a tilt series at each time. Figures $2 \mathrm{a}-\mathrm{c}$ show the atomic models of the same nanoparticle with an accumulated annealing time of 9, 16 and 26 minutes, respectively. A fraction of the surface and sub-surface atoms were re-arranged to form an $\mathrm{L} 1_{0}$ phase, but the Pt-rich core of the nanoparticle remained the same (Figs. 2d-f), which is evident by comparing the same internal atomic layers along the [010] direction (Figs. 2g-i). These observations represent, for the first time, the experimental demonstration of determining the structure and dynamics of materials at 4D atomic resolution. Our experimental results on the early stage nucleation of the $\mathrm{L} 1_{0}$ FePt phase can expand our understanding of the critical conditions and requirements to make superior magnetic recording media and catalysts based on binary alloys. 4D AET allows the capture of the atomic motion in materials in 4D, which is currently not accessible by any other experimental methods [8].

\section{References:}

[1] J. Miao et al., Science 353, (2016), p. aaf2157.

[2] D. J. Bacon \& D. Hull (eds), "Introduction to Dislocations", 5th edn (Butterworth- Heinemann, 2011).

[3] A. A. Kelly \& K. M. Knowles, "Crystallography and Crystal Defects", 2nd edn (John Wiley \& Sons, 2012).

[4] M. C. Scott et al., Nature 483, (2012), p. 444-447.

[5] C. C. Chen et al., Nature 496, (2013), p. 74-77. 
[6] R. Xu et al., Nat. Mater. 14 (2015), p. 1099-1103.

[7] Y. Yang et al., Nature 542 (2017), p. 75-79.

[8] J. Zhou et al., arXiv:1807.10709 (2018).

[9] This work was supported by STROBE: NSF STC (DMR-1548924), US DOE (DE-SC0010378), the NSF DMREF (DMR-1437263), and the Molecular Foundry (US DOE DE-AC02-05CH11231).
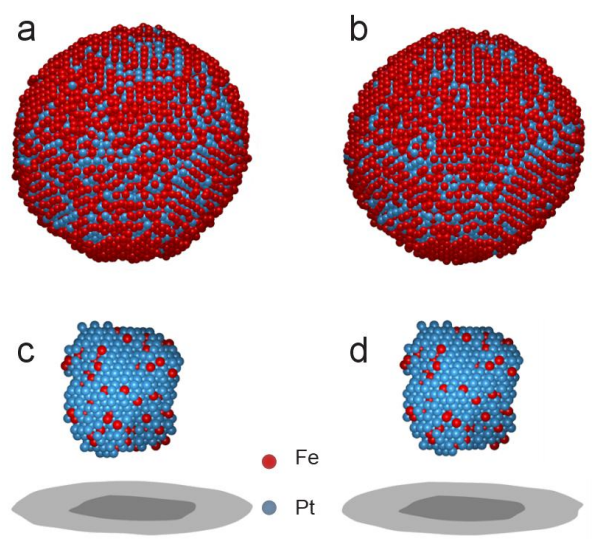
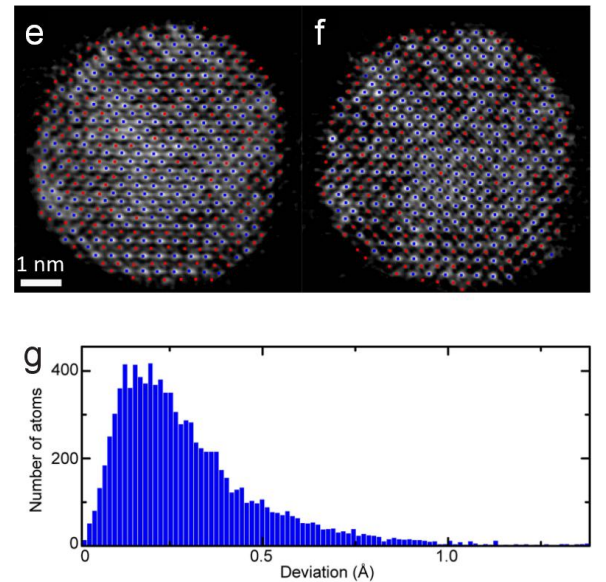

Figure 1. Consistency check of the AET measurements. a and b, 3D atomic models of an FePt nanoparticle, obtained from two independent experimental measurements. c and d, Pt-rich cores cropped from the atomic models shown in (a) and (b), respectively. e and $\mathbf{f}$, The same atomic layer of the nanoparticle along the [010] direction. g, Histogram of the deviation of the common atoms between the two independent measurements.
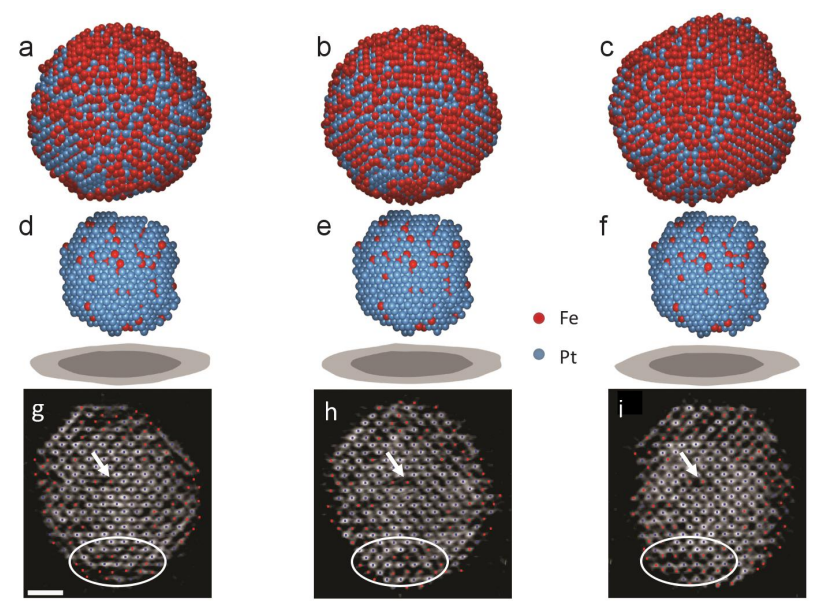

Figure 2. Capturing 4D atomic motion and nucleation dynamics with AET. a-c, 3D atomic models (Fe in red and Pt in blue) of another FePt nanoparticle at different annealing times. d-f, The Pt-rich core of the nanoparticle. g-i, The same internal atomic layer of the nanoparticle along the [010] direction at the three annealing times.(Fe in red and Pt in blue; Scale bar: $1 \mathrm{~nm}$ ). 